\title{
A Flight Demonstration of Plasma Rocket Propulsion
}

\author{
Andrew Petro, Advanced Space Propulsion Laboratory, NASA Johnson Space Center, \\ Houston, TX, Franklin Chang-Diaz, Advanced Space Propulsion Laboratory, NASA \\ Johnson Space Center, Houston, TX, Mark Carter, Oak Ridge National Laboratory, \\ $T N$, William Schwenterly, Oak Ridge National Laboratory, TN, Michael Hitt, \\ Lockheed Martin - Michoud Space Systems, LA, Joseph Lepore, SAIC, Houston, TX
}

\begin{abstract}
The Advanced Space Propulsion Laboratory at the . NASA Johnson Space Center has been engaged in the development of a variable specific impulse magnetoplasma rocket (VASIMR) for several years. This type of rocket could be used in the future to propel interplanetary spacecraft and has the potential to open the entire solar system to human exploration. One feature of this propulsion technology is the ability to vary its specific impulse so that it can be operated in a mode that maximizes propellant efficiency or a mode that maximizes thrust. Variation of specific impulse and thrust enhances the ability to optimize interplanetary trajectories and results in shorter trip times and lower propellant requirements than with a fixed specific impulse.
\end{abstract}

In its ultimate application for interplanetary travel, the VASIMR would be a multi-megawatt device. A much lower power system is being designed for demonstration in the 2004 timeframe. This first space demonstration would employ a 10-kilowatt thruster aboard a solar powered spacecraft in Earth orbit. The 10-kilowatt VASIMR demonstration unit would operate for a period of several months with hydrogen or deuterium propellant with a specific impulse of 10,000 seconds.

\section{Introduction}

Preparations are underway for the first space demonstration of the VASIMR technology. Although significant testing of the thruster will occur on Earth in vacuum chambers, a space demonstration will increase confidence in the operation of the thruster in the space environment over an extended period of time. The test will also allow for measurements of thruster performance and observations of the thruster-induced environment around the spacecraft. The demonstration is proposed as part of the Radiation and Technology Demonstrator (RTD) mission to fly in the 2004 timeframe.

In the flight demonstration, a ten-kilowatt VASIMR engine on a solar-powered spacecraft will be operated over a period of several months. The spacecraft will be deployed from the Space Shuttle at an altitude of 400 kilometers. From there the spacecraft will be propelled in a spiral trajectory to a nearly geosynchronous orbital altitude. The spacecraft will carry a VASIMR engine and also a 10-kilowatt Hall-effect thruster. Since the spacecraft is solar powered and has very limited power storage capacity, these electric propulsion thrusters will operate only during the sunlit portions of the orbit.

The two electric propulsion thrusters will be demonstrated separately but together they will also accomplish a scientific mission to measure the natural radiation environment in Earth orbit. The spacecraft will carry several microsatellites that will be deployed sequentially as the spacecraft gains altitude resulting in a constellation of microsatellites that can make simultaneous radiation measurements at distinct locations. A conceptual design of the RTD spacecraft is shown in Figure 1.

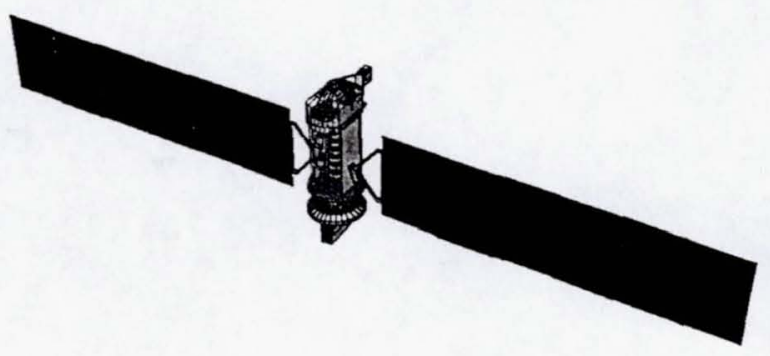

Figure 1: RTD spacecraft conceptual design.

For simplicity in the first space demonstration, the 10kilowatt VASIMR will not employ the variable specific
Copyright 102000 by the American Institute of Aeronautics and Astronautics, Inc. No copyright is asserted in the United States under Title 17, U.S. Code. The U.S. Government has a royalty-free license to exercise all rights under the copyright claimed herein for Government Purposes. All other rights are reserved by the copyright owner. 
impulse feature. The thruster will have a fixed specific impulse of 10,000 seconds which is significantly higher than current electric propulsion systems which typically have a specific impulse of less than 2,000 seconds. The 10-kilowatt VASIMR will produce a thrust of approximately 0.1 Newton and consume 1 milligram of propellant per second, which equates to 17 kilograms in six months of continuous operation.

The VASIMR engine to be flown in this demonstration will use hydrogen or deuterium propellant. To ensure that the spacecraft meets Space Shuttle payload bay . safety requirements, the propellant will be carried to orbit in a separate Space Shuttle tank pallet and transferred to the spacecraft tank after achieving orbit. This approach also results in a lighter and less expensive spacecraft tank. The transfer of cryogenic hydrogen or deuterium will itself be an additional flight demonstration of a technology of great interest to those planning future planetary exploration missions.

Thermal control is the most significant engineering challenge in the design of this initial flight version of the rocket. The cryogenic propellant must be maintained with very little boiloff for many months. There is a superconducting electromagnet that will need to be maintained at cryogenic temperatures in order to operate properly. These super-cold elements are in close proximity to the plasma so a combination of compact insulation and passive and active heat transport techniques must be employed.

This mission will be the first demonstration of the VASIMR propulsion technology in space. At ten kilowatts, it will be a relatively low-power demonstration but will blaze the trail for much higherpowered systems that will lead to full-scale electric propulsion for practical robotic and human space flight throughout the solar system.

\section{Thruster Configuration}

The thruster assembly is composed of an inner ceramic tube in which the neutral propellant is injected and ionized within a larger metallic-lined tube supporting the radio frequency antennas, which ionize the gas and heat the plasma. These tubes are surrounded by electromagnet coils that provide a magnetic field that constrains the flow of the plasma and forms the magnetic exit nozzle. The tubes, antennas, and surrounding magnet assembly form the core of the thruster. The two tubes intercept heat generated by the plasma and RF antennas and shield the low-temperature magnet assembly. The design will include a method for transporting heat in the tubes out to radiator surfaces that form the outer enclosure of the rocket assembly. A cross-sectional view of the thruster core is shown in Figure 2.

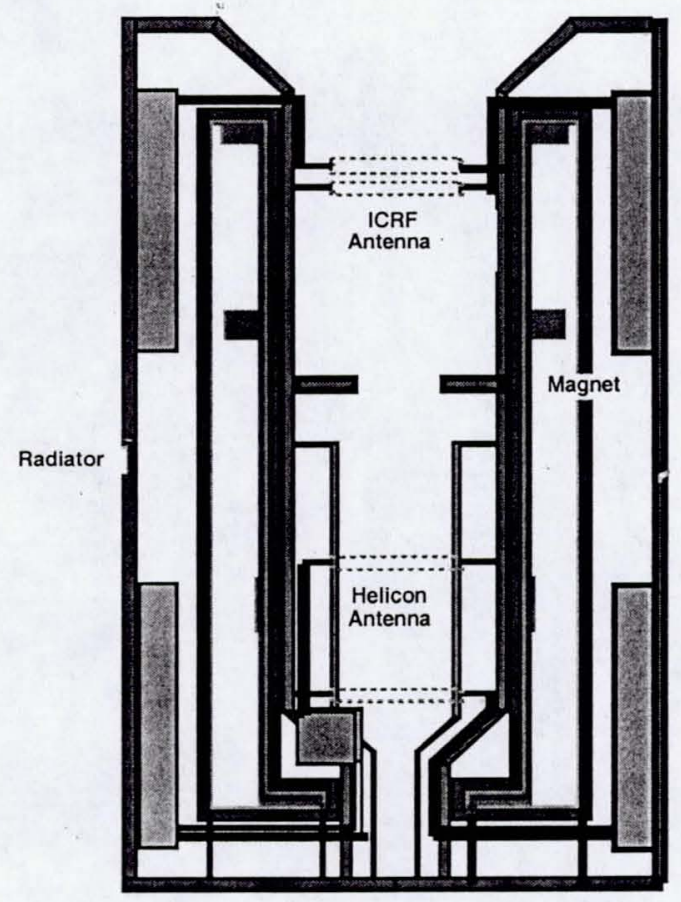

Figure 2: Ten-kilowatt VASIMR thruster core.

The spacecraft will be flown in Earth orbit in an orientation that maintains the engine thrust vector along the velocity vector while orienting the solar arrays toward the sun. The solar arrays have one gimbal axis and so the spacecraft will be rotated about its roll axis in order to keep the solar arrays perpendicular to the solar flux at all times during the lighted portion of the orbit. This means the same two sides of the spacecraft and two sides of the attached thruster will generally view deep space throughout the orbit.

The cylindrical thruster core will be located within the rectangular enclosure of the rocket. The enclosure is designed to minimize the surface area that is exposed to solar flux while maximizing the surface area that is exposed to deep space. The sides of the enclosure facing deep space will serve as a thermal radiator and the other sides will be made as reflective as possible to minimize solar heating of the thruster. The thruster core and rectangular enclosure are located behind the propellant tank. The radio-frequency amplifiers and other electronic components will be located within the rectangular enclosure on the inner surface of the radiator panels.

The rectangular enclosure has dimensions of $60 \mathrm{~cm}$ by $100 \mathrm{~cm}$ by $200 \mathrm{~cm}$. The diameter of the main tube is a little over $20 \mathrm{~cm}$ and its length is about $80 \mathrm{~cm}$. The 
diameter of the magnet assembly container is $40 \mathrm{~cm}$. An isometric, cutaway view of the thruster is shown in Figure 3.

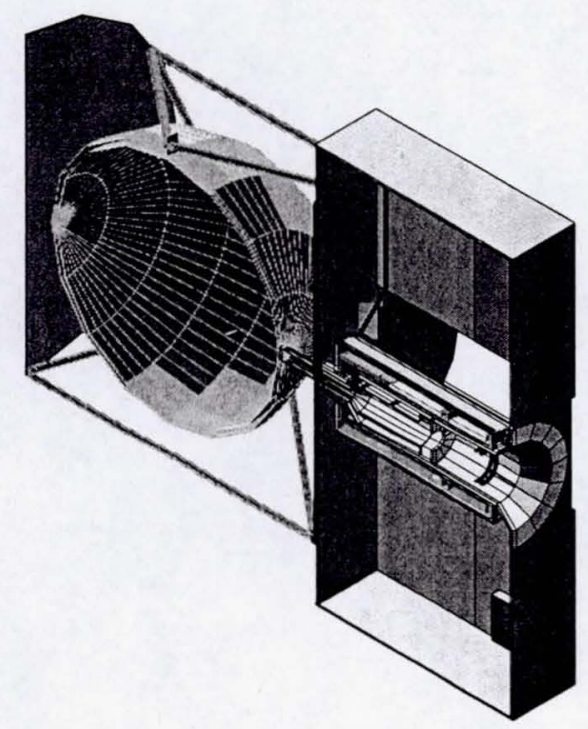

Figure 3: Isometric, cutaway view of ten-kilowatt VASIMR thruster.

\section{Propellant System}

The ten-kilowatt VASIMR thruster will use hydrogen or deuterium as the propellant. The propellant storage system described here has been designed for hydrogen but the system would be similar if deuterium is used. The propellant tank is sized to hold up to 50 kilograms of supercritical hydrogen at a pressure of $1380 \mathrm{kPa}(200$ psi) and a temperature of $28 \mathrm{~K}$. Supercritical hydrogen exists as a single-phase fluid. At the planned usage rate of one milligram per'second, the rocket will only consume about 17 kilograms of propellant in six months of continuous operation so the tank as currently designed would contain a substantial surplus of propellant. The aluminum tank is spherical with a diameter of 1.2 meters and an average thickness of approximately two millimeters. The tank will be covered with a 2 to 3 centimeter thickness of multilayer insulation.

Since the spacecraft will be delivered in the Space Shuttle there are concerns about payload bay safety associated with the hydrogen propellant. An approach has been developed to satisfy hydrogen safety issues and this approach provides several other benefits. The approach is to carry the existing Extended Duration Orbiter (EDO) pallet as a payload in the Shuttle along with our demonstration spacecraft. The EDO pallet was developed to carry additional hydrogen and oxygen reactants to supply the Shuttle's fuel cells during long orbital missions. The EDO pallet is not needed during normal Shuttle missions so we can use the hydrogen tanks to carry the propellant needed for the VASIMR.

Once in orbit, the hydrogen can be transferred from the pallet tanks to the VASIMR tank. This means that the hydrogen can be launched in tanks that have already been certified and flight-proven. Great expense will be avoided by not having to design and certify the new VASIMR tank for launch with hydrogen in the Space Shuttle. An illustration of the VASIMR tank and the EDO pallet is provided in Figure 4.
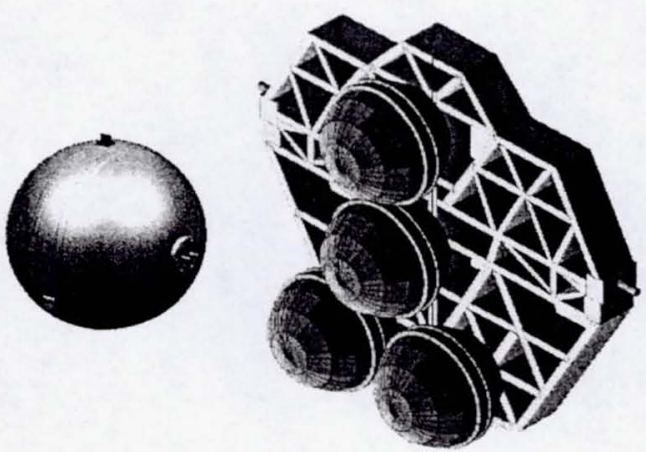

Figure 4: VASIMR tank concept and EDO pallet.

The in-space transfer of cryogenic hydrogen has value in its own right as a demonstration of a process that may be very important in future space operations. Also, since the VASIMR tank will not carry any cryogenic fluid during launch, it will not require a heavy vacuum jacket and does not have to be designed to carry any significant fluid load during ascent. The EDO pallet normally carries four hydrogen tanks, each with 42 kilograms of hydrogen. Three tanks should be sufficient to fill the VASIMR tank including the quantity needed for chill-down. The fourth tank provides margin.

It is expected that a sufficient quantity of hydrogen can be stored in space for the duration of the demonstration mission using only passive thermal insulation. Before entering the thruster, the hydrogen will pass through a network of tubes that will provide some cooling for the superconducting electromagnet. 


\section{Radio-Frequency System}

The radio-frequency system uses electrical power to ionize and heat the propellant; converting cold neutral gas into hot plasma. The thruster contains a helicon antenna surrounding a propellant injection tube where ionization takes place. Once ionized, the plasma flows toward the rocket outlet, following axial magnetic field lines. Downstream from the helicon antenna, the plasma flows through the ion-cyclotron-resonanceheating (ICRH) antenna that adds energy to the plasma. The extremely hot plasma follows the diverging magnetic field lines of the nozzle section. The plasma particles gradually break away from the magnetic field lines and leave the rocket with primarily axial velocity, thus generating thrust.

The helicon and ICRH antennas are each composed of two circular rings that allow them to generate phaseshifted electrical fields. These electrical fields, working in conjunction with the surrounding magnetic field, ionize the gas and heat the plasma. A set of oscillators with amplifiers feed radio-frequency signals to each antenna along flat-strip transmission lines. Each antenna is grounded to the conducting wall of the main tube to provide a return path for the signals.

Each amplifier unit contains two solid-state amplifier components and is designed to operate with an input power of up to one kilowatt and an efficiency of approximately 80 percent. Each antenna is fed by a set of three amplifier units connected through a combiner circuit. An antenna will be able to function even if one of its three amplifier units fails. With three amplifier units for each antenna segment there would be a total capacity of twelve kilowatts but the system will operate at a lower power level since a supply of only ten kilowatts is expected. The antennas, transmission lines, and amplifiers are shown in their proper positions in figure 5 .

\section{Eleetromagnet System}

The VASIMR requires a magnetic field that is tailored to provide proper containment of the plasma as it flows through and out of the rocket. Local magnetic field strength is also a factor in the proper interaction of the antenna fields and the plasma. The magnetic field is generated by a series of electromagnet coils that surround the tube and antenna assembly. The maximum axial and radial magnetic field strengths are respectively 0.8 and 0.6 tesla. The current design of the coils and resulting field lines are shown in figure 6.

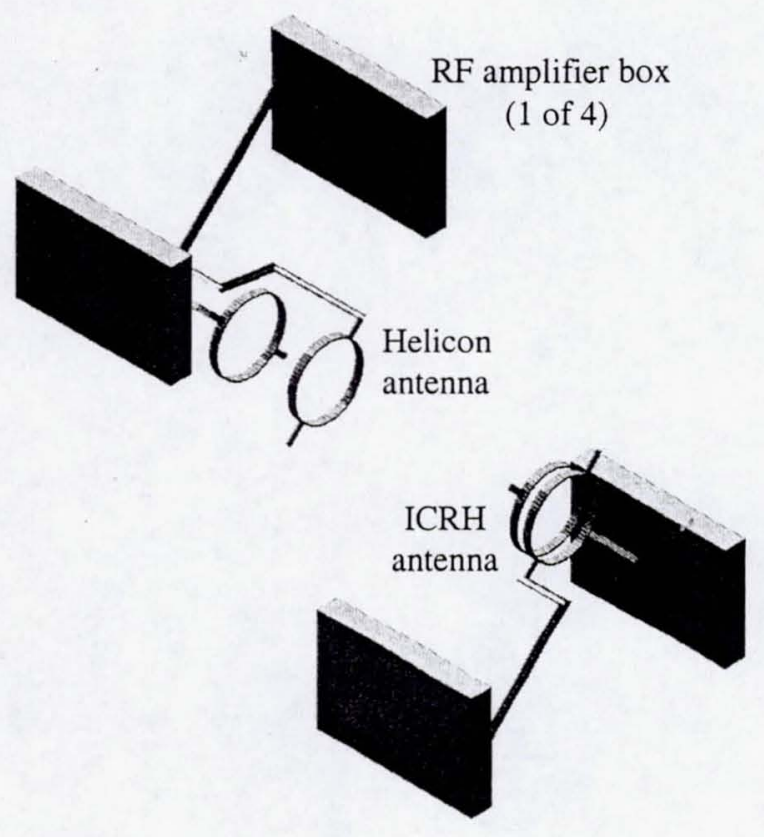

Figure 5: Radio-frequency system.

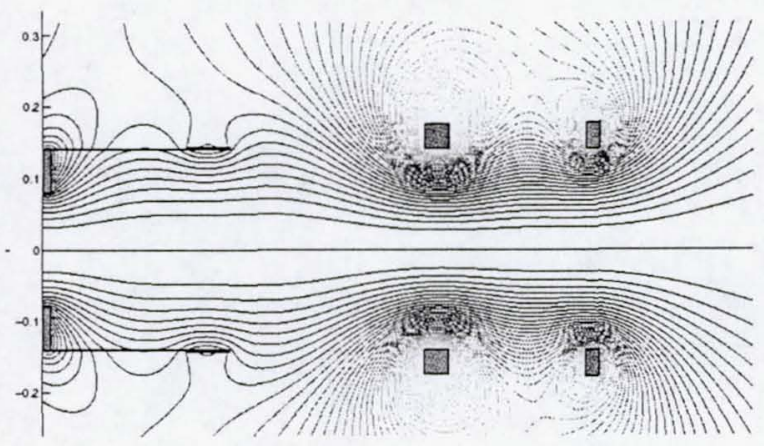

Figure 6: Electromagnet coils and field lines.

\section{Thermal Control}

The VASIMR presents a series of interesting thermal control challenges. There is a need to store cryogenic propellant and to maintain the superconducting magnet at cryogenic temperatures. At the same time there are electronic amplifiers which generate some waste heat, antennas and transmission lines which will experience heating due to the current they carry, and extremely high-temperature plasma at the core of the rocket. The spacecraft will experience a constantly changing thermal environment as it orbits the Earth in a spiral of increasing altitude. The systems that maintain acceptable thermal conditions need to be compact, 
lightweight, inexpensive, and able to operate reliably for many months. For this reason passive systems will be used as much as possible.

The preliminary strategies and options for thermal control are outlined below for each major system.

Propellant Tank: The hydrogen or deuterium will be stored in a simple single-walled tank which is covered with multilayer insulation. There will be only three structural attach points on the tank. Materials with low thermal conductivity will be used at the structural interfaces and as much as possible in the fluid lines connecting the tank with the thruster. Flat circular thermal shields will be installed above and below the tank to intercept heat radiated from the thruster assembly and the RTD spacecraft. There will be some degree of heat leakage but the amount of excess propellant being carried should be able to sustain a low but continuous boiloff rate.

Radio-Frequency Systems: The amplifiers and other electronics will be mounted on cold-plates which will be cooled either by direct conduction to the radiator surface or by a system of heat pipes or a pumped fluid loop. The tranmission lines and antennas conduct their current along the outer surface. These components will be designed as integral heat pipes or with built-in fluid lines connected to a pumped-loop, unless a material can be found that has sufficient thermal conductivity to provide completely passive heat transfer.

Superconducting Electromagnet: The spool on which the superconducting material is wound will be cooled to a temperature of nearly $35 \mathrm{~K}$ by tubes carrying cryogenic propellant on its way from the storage tank to the thruster inlet. The magnet and spool will be surrounded by a thermal shield which will be maintained at a temperature near $80 \mathrm{~K}$. It is hoped that this can be done through passive insulation but if necessary a cryogenic cooler device will be used. The shield limit of $80 \mathrm{~K}$ is the highest temperature that the magnet and spool can view and still maintain its temperature limit using the normal one milligram per second propellant flow rate for cooling.

The magnet shield intercepts heat coming from the thruster core on the inside and from the surrounding structure and the space environment on the outside. The entire surface of the shield would be covered with multilayer insulation. All of the voids between the magnet spool, shield, and the thruster will be vented to vacuum so that heat transfer is limited to radiation except for the necessary structural attachments which will be made of materials which minimize heat conduction.
Thruster Core: The main tube and the smaller tube within the helicon antenna will intercept the majority of any heat radiated by the plasma. These tubes will employ conduction to tranport the heat along their length and out to radiator surfaces which face deep space. Heat pipes or pumped-loop fluid lines may be used to augment the thermal conduction.

Radiators: A prominent feature of the 10-kilowatt VASIMR thruster is the rectangular enclosure which forms the thermal shield and radiator surfaces. These radiators will be used to reject the heat from the thruster core, the antennas, and amplifiers. Since the thruster core may be maintained at a higher temperature than the electronic components, the radiator panels may be split into higher temperature and lower temperature regions. There will be a window in the side of each radiator panel so that the magnet shield will have a view to space for direct heat rejection rather than viewing the inside surface of the radiator panels. The option may also exist to transport some heat from the VASIMR thruster to radiator panels on the RTD spacecraft.

Thermal modeling and analysis has been performed to aid in the design of the thruster. An example of the analysis results is shown in figure 7 . As a worst-case assumption, waste heat values as high as 6000 watts $(60 \%)$ have been used in the analysis although the overall performance of the system is expected to be much better. This work will allow for the evaluation of a range of thermal control strategies including highconductivity materials, heat pipes, and pumped fluid loops. The ultimate design will incorporate passive systems to the greatest extent possible.

\section{Diagnostics and Control}

In order to observe the characteristics of the plasma within the rocket there will be a set of photodiodes, an interferometer, and possibly a video camera. Overall system performance and health will be monitored with sensors for voltage, current and magnetic field strength, and numerous temperature sensors. There will also be temperature, pressure, and flow sensors for the propellant supply system and position sensors for any valves required in the propellant and thermal control systems.

Some command capability will be provided for radiofrequency system operation, magnet activation, propellant flow, and possibly the operation of some diagnostic devices. Comands and telemetry will be routed through the communications system on the RTD spacecraft. 


\section{Acknowledgements}

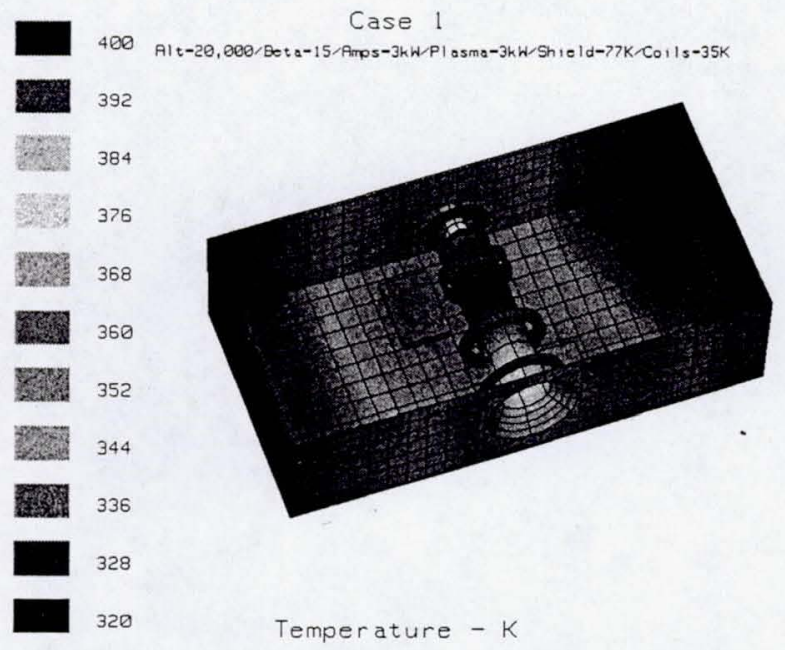

Figure 7: Example of thermal modeling results.

\section{Summary}

The current mass estimate for the 10-kilowatt VASIMR is $150 \mathrm{~kg}$ for the thruster and enclosure, 68 kilograms for the propellant tank, plus 50 kilograms of propelllant for a total wet mass of 268 kilograms. The mass estimate includes growth margin and many conservative assumptions have been made in the design. The potential exists to reduce this mass estimate during further design refinement.

Significant work remains to be done in the refinement of the design, investigation of material options, and in particular, in the analsysis and design of the thermal control systems. Ongoing measurements of heat loads in the VASIMR laboratory experiment may shed more light on thermal conditions within the thruster and allow for major design improvements.

While work continues toward the first space demonstration of magnetoplasma propulsion, studies are underway for higher-power system demonstrations and the eventual application of the technology in highspeed exploration missions for robots and people to Mars and the planets beyond. This technology may also represent an early step toward propulsion systems which will extend our reach to distant stars.
In addition to the co-authors, the following individuals contributed to this work: Andrew Ilin and Jared Squire, Advanced Space Propulsion Laboratory; Rick Goulding, Wally Baity, Glen Barber, and Dennis Sparks, Oak Ridge National Laboratory; Roger Bengston, University of Texas; Charles Camarda, Gene Ungar, and Howard Wagner, Johnson Space Center; and Derek Townsend and Steve Jones, Lockheed Martin - Michoud Space Systems. 